\title{
TEORÍA Y PRÁCTICA DE LA CORTE PENAL INTERNACIONAL A TRAVÉS DE UN PROCESO SIMULADO
}

\section{THEORY AND PRACTICE OF THE INTERNATIONAL CRIMINAL COURT THROUGH A SIMULATED PROCESS}

Juan Luis Jiménez Ruiz (Universidad de Jaén) ${ }^{1} 2$

\section{Resumen:}

El Instituto Iberoamericano de La Haya para la Paz, los Derechos Humanos y la Justicia Internacional organiza al auspicio de la Corte Penal Internacional, y con la colaboración de otras entidades, un ejercicio anual de simulación judicial previsto para alumnado de los diferentes ciclos universitarios. Los roles que adoptan los participantes en el concurso son los propios a los que en la realidad desempeñarían procesalmente la Fiscalía, la Representación legal de las víctimas y la Defensa. Se trata pues de uno de los concursos de simulación judicial más relevantes, entre los de su clase, en el panorama internacional, pues supone introducir al alumnado en las competencias propias requeridas en órganos jurisdiccionales internacionales, entre los que se encuentra la Corte Penal Internacional. Este ensayo expone la experiencia relativa a la preparación, participación y repercusión, en el equipo que representó a la Universidad de Jaén, en la VII edición del Concurso de Simulación, que tuvo lugar en La Haya, durante el mes de mayo de 2019.

Palabras clave: Derechos humanos. Corte Penal Internacional. Simulación judicial internacional.

Códigos JEL: A22, I23.

\begin{abstract}
:
The Ibero-American Institute of The Hague, for Peace, Human Rights and International Justice, an organization under the auspices of the International Criminal Court, an annual judicial simulation exercise planned for students of different university cycles. The roles adopted by the participants in the contest are those that actually prosecute, prosecute and prosecute. It is one of the most relevant judicial simulation competitions of its kind in the international arena, as it involves the introduction of students into the competencies required in international jurisdictional courts, among which is the International Criminal Court. This work exposes the experience of the preparation and the impact of the team that represents the University of Jaén in the VII edition of the Simulation Process that took place in, La Haya, in 2019.
\end{abstract}

\footnotetext{
1 jlruiz@ujaen.es, Universidad de Jaén.

${ }^{2}$ La actividad que se presenta ha sido desarrollada en el marco del "Semillero de investigación, sensibilización y cooperación jurídica en materia de Derechos Humanos” (D Juana Pérez, Da $M^{\mathrm{a}}$ del Carmen Castilla, Da Ana Paula Martín, D. Alejandro Araque, Da Isabel Trillo Espejo).

Recibido 10 de octubre de 2019. Aceptado 2 de diciembre de 2019.
} 
Key words: Human rights. International Criminal Court. International Judicial Simulation. JEL Codes: A22, I23.

\section{PLANTEAMIENTO}

La tutela de los derechos humanos/fundamentales no se agota jurídicamente en el ámbito interno de los Estados. Su defensa es susceptible de ser ejercida, entre otros múltiples mecanismos, en la jurisdicción internacional, sea en su faceta regional o universal.

Dichos ámbitos requieren para los actores procesales que participan en los procesos judiciales internacionales una alta preparación, además de la adquisición de competencias particulares, imprescindibles para afrontar con éxito la tarea.

Las premisas anteriores determinan el objetivo principal de la acción a presentar, que es el de introducir al alumnado de la Universidad de Jaén, en concreto al procedente del Doble Grado en Administración y Dirección de Empresas y Derecho, y del Grado en Derecho, a través de los ejercicios de simulación judicial internacional, en la actuación procesal propia de los procesos internacionales; en concreto los circunscritos a la Corte Penal Internacional (CPI).

Al objetivo principal se anudan objetivos, cuya naturaleza secundaria, no resta valor a su relevancia. Pues con esta acción se persigue ampliar por un lado el conocimiento que el alumnado recibe en materia de promoción, protección y tutela de los Derechos humanos/fundamentales, tanto en perspectiva teórica como práctica. Por otro, se busca también alumbrar al alumnado sobre posibles vías laborales propias de Organizaciones Internacionales y sus instituciones. Ello a través de la participación en un ejercicio de simulación judicial que finalmente culmina, tras diversas fases, escenificando el proceso mismo en la sede de la Corte Penal Internacional ${ }^{3}$.

Salta a la vista que son múltiples los retos a afrontar por parte del alumnado, y su preparador, para alcanzar el objetivo señalado. Entre otros, considérese: i) Se trata de la preparación de personas que aún no han culminado sus estudios técnicos en Derecho (Grado). ii) Se requieren conocimientos legales, sustanciales y procesales, específicos de la jurisdicción internacional. iii) Deben demostrar competencias personales que les permitan afrontar el reto, tales como una alta motivación, dinamismo, clara oralidad, además de una correcta puesta en escena.

Sintetizo esta introducción afirmando que este trabajo aborda el tracto general de la preparación del concurso indicado, tratando de contemplar desde una múltiple perspectiva las repercusiones obtenidas tras la participación en el mismo, por parte del equipo de la Universidad de Jaén, ante y durante una primera experiencia ante la CPI (VII edición 2019).

\section{LA CORTE PENAL INTERNACIONAL: ALGUNAS NOTAS}

La CPI ${ }^{4}$ supone, en perspectiva jurídica, un muy trascendente -tal vez sin parangón- hito jurídico en la historia de la humanidad. Por primera vez la Sociedad Internacional cuenta con un

\footnotetext{
${ }^{3}$ Puede visionarse la final de la VII Edición del Concurso CPI de Simulación judicial ante la CPI (2019), a través del enlace: https://www.youtube.com/watch?v=DQWMIjVa6yc .

${ }^{4}$ Para conocer la CPI a través de una fuente directa consúltese su web oficial, a través del enlace: https://www.icccpi.int/
} 
tribunal penal permanente previsto para conocer las más graves violaciones de derechos humanos acaecidas en aquellos Estados que son parte del Estatuto de Roma (Vallejo Peña, 2016, p. 20). Estatuto que constituyó y puso en marcha la CPI en el año 2002. La importancia estriba en que la CPI rompió con los tan polémicos, como hasta entonces necesarios, tribunales penales internacionales ad hoc. Tribunales que eran creados tras el conflicto y que también aplicaban normativa creada ex post para enjuiciar los sucesos ocurridos.

Por tanto la CPI es la primera jurisdicción internacional de materia criminal, permanente, creada por la humanidad, hábil para juzgar a aquellas personas naturales responsables de los crímenes ${ }^{5}$ más abominables cometidos con posterioridad a la entrada en vigor de su Estatuto. En concreto sustancia crímenes contra la humanidad, de genocidio, crímenes de guerra y, -tras la Conferencia de Revisión del Estatuto de Roma (Kampala 2010)-, también el crimen de agresión, aunque este último solo en el caso de los Estados que lo han ratificado, como el caso de España, la revisión señalada.

La CPI surgió por impulso de las Naciones Unidas ${ }^{6}$, aunque de modo independiente a dicha organización, en virtud del Estatuto de Roma, de 17 el julio de 1998, y entró en vigor el 1 de julio de 2002. Quedando desde entonces constituida en La Haya, Países Bajos. Este hecho rompió con la histórica tradición de crear tribunales penales especiales post-conflicto (Ambos, 1998, p. 1697) tales como los que se configuraron tras la Segunda Guerra Mundial en los Juicios de Núremberg y en el Tribunal Penal Militar Internacional para el Lejano Oriente (Procesos de Tokio). Surgieron otros con posterioridad el Tribunal Penal Internacional para la ex-Yugoslavia, el Tribunal Penal Internacional para Rwanda; las Salas Especiales en los Tribunales de Camboya, el Tribunal Especial para el Líbano, el Tribunal Especial Residual para Sierra Leona y el Mecanismo Residual Internacional de los Tribunales Penales. Incluso en la actualidad (2019) se encuentran intentos de constituir nuevos y particulares Tribunales internacionales. Véase a los efectos señalados el Proyecto de la Corte Penal Latinoamericana y del Caribe contra el Crimen Transnacional Organizado (COPLA) ${ }^{7}$ que promueven algunos Estados latinoamericanos.

A través de dichas instancias jurisdiccionales de naturaleza internacional y penal es como la humanidad ha exigido, a partir del S. XX, su responsabilidad, a los perpetradores de los crímenes, cuya naturaleza, el propio Estatuto CPI señala en su Preámbulo, al afirmar: “Teniendo presente que, en este siglo, millones de niños, mujeres y hombres han sido víctimas de atrocidades que desafían la imaginación y conmueven profundamente la conciencia de la humanidad,”.

Cabe aclarar que si bien la CPI se ha ido afianzando a lo largo de las dos décadas de historia, afronta muy importantes retos de cara a su real y efectiva consolidación en la Sociedad Internacional, pues se encuentra sitiada por un fuerte estatalismo. Amenaza contemporánea de prácticamente la totalidad de las organizaciones internacionales (Annello, 2013, p. 43). En el caso de la CPI la posición hostil de algunos Estados roza la beligerancia, sobre todo en aquellos que no han ratificado, o se han retirado, de su Estatuto, tales como Estados Unidos, Rusia, China o India, por citar los casos más llamativos en atención a la relevancia de los Estados de los que se trata. Lo anterior contrasta con el número de Estados que o bien han firmado su Estatuto (139 Estados), o bien son Estados miembros del mismo (124 Estados), (Vallejo Peña, 2016, p. 23).

\footnotetext{
${ }^{5}$ Consúltese el Estatuto de Roma, art. 5.

${ }^{6}$ Véase el Acuerdo de relación entre la Corte Penal Internacional y las Naciones Unidas, Nueva York, 4 de octubre de 2004. En vigor 04 de octubre de 2004.

${ }^{7}$ Amplíese sobre COPLA a través de su web: https://www.coalicioncopla.org/.
} 
Una perspectiva, de entre las infinitas válidas, para el jurista en torno a los Tribunales de jurisdicción internacional puede ser la relativa a que en realidad éstos no son ni más, ni menos, que órganos jurisdiccionales, previstos por un sistema jurídico, lógicamente, determinado. En consecuencia a ellos los juristas deben acudir pues son miembros fundamentales del engranaje técnico que les da soporte en el ejercicio de sus competencias y son quienes, en definitiva, permiten su funcionamiento.

Lo anterior determina por fuerza que los Tribunales de jurisdicción internacional presentan múltiples particularidades que se vinculan a sus rasgos esenciales, entre otros: son creados en los sistemas internacionales, bien como instituciones de una Organización Internacional (Corte Internacional de Justicia (CJI), Tribunal Europeo de Derechos Humanos, Corte de Justicia de la Unión Europea...) o bien, como una Organización Internacional independiente, como es el caso de la CPI. Sus competencias territoriales pueden tener vocación universal (CIJ o el Tribunal Internacional de Derecho del Mar) o regional, referidas éstas a los sistemas internacionales regionales (Europeo, Africano, Americano, Asiático). La legitimación subjetiva también los distingue, pues se encuentran aquellos ante los que sólo pueden acudir (activa y pasivamente) los sujetos internacionales (Estados y Organizaciones Internacionales) y otros admiten la legitimación del individuo mediante su presentación como parte en el proceso (TEDH) y/o procesado en el mismo (CPI).

En efecto la CPI tiene competencia para juzgar, después de su entrada en vigor, a cualquier persona natural que proceda de un Estado parte de su Estatuto, o bien que su Estado haya reconocido la competencia de la Corte. Los individuos han de ser mayores de dieciocho años, y no pueden objetar, -ni la CPI reconocer-, preeminencias personales o políticas de ningún tipo, tales como inmunidades o inviolabilidades (Olásolo et al., 2016, p. 257).

Se finaliza este apartado señalando el carácter complementario de la jurisdicción de la CPI sobre los Estados miembros del Estatuto de Roma y es que son los Estados en sus respectivas jurisdicciones nacionales quienes tienen el deber de perseguir en su territorio, y en determinados casos también fuera de él (previsiones de justicia universal), los hechos que en última instancia perseguirá la CPI. Esto concuerda con el no reconocimiento de preeminencias personales indicada anteriormente $\mathrm{y}$ es que, en virtud de éstas, muchos Estados se ven limitados e incluso imposibilitados de perseguir a los/as responsables de las atrocidades perseguidas por la CPI entre los límites de su soberanía. Circunstancia que en última instancia justifica la creación, existencia y consolidación de la CPI.

\section{LA SIMULACIÓN JUDICIAL ANTE LA CORTE PENAL INTERNACIONAL}

Las pautas precisas de la organización del Concurso aparecen publicadas en la web del Instituto Iberoamericano de La Haya ${ }^{8}$, donde se remite para conocer todo con la debida precisión. En dicho portal aparece publicado anualmente la convocatoria, así como las instrucciones y documentación necesarias para participar en el proceso.

En cada convocatoria la organización propone un caso ficticio que los participantes deben abordar desde una triple perspectiva: Fiscalía, Representación legal de las víctimas y Defensa del procesado. Ello implica adoptar roles muy diversos, incluso opuestos en su discurso, en un mismo equipo, y fundamentar así jurídicamente de manera solvente cada una de estas tres posiciones. Lo

\footnotetext{
${ }^{8}$ Disponible, a través del enlace: https://www.iberoamericaninstituteofthehague.org/es/actividades/concurso-desimulacion-judicial-sobre-la-cpi/163-concurso-cpi.
} 
que requiere un alto grado de trabajo e implicación por parte de todos los participantes, en la medida en que tendrán que investigar el caso propuesto, sustanciarlo y defenderlo de manera coherente con las normas jurídicas aplicables.

Publicado el caso, los equipos cuentan con algunos meses para su estudio, previos a satisfacer el trámite de la inscripción en el Concurso, trámite que determinará la participación efectiva en el mismo, a través de la entrega de memoriales algunos días después.

El caso a analizar, lógicamente ficticio, -guarda relación con los tipos penales, que desde la esfera internacional puede perseguir la CPI- incluye las cuestiones que específicamente deben ser tratadas por los componentes de los equipos que se presentan al concurso y deben ser abordadas, desde su particular perspectiva por los roles adoptados por Fiscalía, Representación legal de las víctimas y Defensa. Dicho estudio servirá para la presentación de los memoriales que para cada uno de esos roles es preciso consignar.

Reténgase la dificultad intrínseca sobre lo que se acaba de señalar pues de ello resulta que los componentes de un mismo equipo tendrán que abordar el caso desde una triple y antagónica perspectiva. Reflexiónese en torno a la complejidad de analizar el supuesto práctico desde una triple perspectiva (Fiscalía o Representación legal de las víctimas y, posteriormente, desde la perspectiva que debe emplear la Defensa de la persona/s procesada/s).

\section{LA PREPARACIÓN DEL ALUMNADO}

Se especifica que se describe únicamente y de manera básica el proceso de enseñanzaaprendizaje y se obvia por tanto otras tareas importantes e imprescindibles para llevar a cabo esta actividad, tales como la preparación logística, que como se podrá imaginar no ha resultado precisamente sencilla.

La participación en el Concurso requiere la entrega, previa a la inscripción, por parte del equipo, de tres memoriales, uno por cada una de las partes que intervendrán en el proceso: Fiscalía, Representación legal de las víctimas y Defensa. Es su fase escrita. Por lo tanto, se trata de una tarea, fundamentalmente de investigación jurídica, que deben llevar a cabo los participantes del equipo asistidos por su preparador.

En función del nivel de formación de los participantes, es tarea del preparador evaluar el nivel de conocimientos técnicos que demuestran. Considérese que, en el caso del equipo de la Universidad de Jaén, han sido alumnos/as que cursaban $2^{\circ}, 3^{\circ}$ y $4^{\circ}$ de los Grados de Derecho y del Doble Grado en Derecho y ADE. En esos estadios su conocimiento del Derecho es por fuerza muy limitado, y la idea del orden y de los sistemas jurídicos, máxime los internacionales, es muy abstracta. Considérese que algunos de los participantes tuvieron su primer contacto con el Derecho Internacional Público, al tiempo en que preparaban los referidos memoriales. Naturalmente son requeridos también conocimientos propios del Derecho penal y procesal, además de los propios sobre la CPI.

El primer paso consistió en hacer comprender el nivel jurídico y por tanto de trabajo ante el que nos encontrábamos: nivel Internacional General y en concreto ante la Corte Penal Internacional. A partir de ahí debieron involucrarse en una investigación técnica multidisciplinar sobre el caso planteado, a objeto de que les permitiera defender sus posiciones. De este modo fueron madurando las ideas y posicionamientos que técnicamente alcanzaban sobre el caso. Aclaro que la investigación fue llevada directamente por los participantes, siendo tarea del preparador orientarles en sus investigaciones, validando sus resultados y/o corrigiendo posibles desvíos. 
Mediante la presentación de los memoriales concluye la fase escrita del proceso. Comenzando posteriormente la fase oral, que tiene lugar en la ciudad de La Haya (Países Bajos). La fase oral se desarrolla a lo largo de algunos días, en los cuales los equipos comienzan a competir entre sí, adoptando por lo general un rol diverso en cada ocasión. Esta fase se ordena en etapas que deben superar los equipos para alcanzar la final del Concurso. Se señala que para abordar la fase oral del concurso el alumnado de la Universidad de Jaén realizó numerosos ensayos previos, algunos de ellos en entornos formales.

\subsection{Objetivos}

El objetivo principal es que el alumnado comprenda la relevancia y pragmatismo jurídico de los Derechos humanos participando para ello en mecanismos internacionales previstos para su tutela. Lo que ha sido posible en virtud de la participación del equipo de la Universidad de Jaén en el concurso de simulación judicial ante la CPI.

A él se anudan otros objetivos igual de relevantes:

-Alcanzar conocimientos técnicos y prácticos de los estudios de Derecho en el marco, básico, del Derecho internacional público, penal, internacional penal y procesal y trabajar con ellos mediante su integración e interacción.

-Complementar el conocimiento sobre los sistemas internacionales en particular sobre el sistema internacional, en su ámbito universal, y en concreto sobre la Corte Penal Internacional.

-Potenciar la práctica jurídica en los participantes.

-Toma física de contacto con las sedes, instalaciones y personal (Función Pública Internacional) de estos entes internacionales. Durante la estancia en La Haya el equipo de Jaén pudo tomar contacto con el Sr. Presidente de la Corte Internacional de Justicia (máximo órgano jurisdiccional de las Naciones Unidas), Sr. Philippe Couvreur. Con la magistrada de la CPI, Sra. Da Luz Ibáñez Carranza, con miembros de Europol, Eurojust... También se visitó el Palacio de la Paz, sede de la Corte Internacional de Justicia, y la sede de la Corte Penal Internacional, por citar algunos ejemplos.

-Realización de contactos con equipos de otras Universidades e Instituciones.

-Fomento de capacidades: tales como el trabajo en equipo, coordinación y liderazgo, compañerismo, oratoria, puesta en escena...

\subsection{Competencias, destrezas y capacidades}

Los objetivos fijados determinan entre otras las siguientes competencias:

-Capacidad técnica investigadora: enfocada, al menos, en dos vertientes: una por cuanto a la teoría del Derecho Internacional Público se refiere; otra para la parte especial referida a la CPI. Lo que cristaliza en la presentación de los respectivos memoriales, imposibles de realizar sin el trabajo de análisis previo.

-Dominio multidisciplinar del ordenamiento jurídico y de sus diferentes áreas, en particular las que se adscriben al Derecho público.

-Capacidad de desenvolverse en ambientes laborales complejos (Organizaciones e Instituciones internacionales), lo que requiere gran carga de dinamismo y capacidad de adaptación.

-Capacidad de enfrentarse a un auditorio, llegado el caso de acudir como actor a la final, la Sala de audiencias de la Corte Penal Internacional y a sus jueces/as (reales). 
-Desarrollo de capacidades de autocontrol necesarias para la puesta en escena: tales como oratoria, correcta vocalización y proyección de voz, entonación, cadencia, lenguaje gestual y corporal, control de estrés...

-Capacidad de elaborar dictámenes técnicos, así como de satisfacer los trámites procesales propios de la instancia ante la que se actúe.

\subsection{Retos y dificultades}

Han sido múltiples las eventualidades que se han debido superar para lograr la efectiva participación en el Concurso.

Por un lado, ha sido preciso complementar el trabajo de los participantes y orientarles ante y durante el Concurso, en la medida en que, tal y como se apuntó al inicio, resultan estudiantes de los Grados de Derecho y del doble Grado en Derecho y ADE, que aún no han superado, siquiera, la etapa de formación técnica, en la que naturalmente se encuentran. Para superar la anterior ha sido fundamental contar y potenciar con la natural motivación que ya presentaban los propios participantes. Considérese que esta acción ha estado vinculada a la voluntaria implicación de los participantes en el proyecto: "Semillero de investigación y sensibilización en materia de Derechos Humanos"9 que inició a trabajar como un proyecto patrocinado por el Vicerrectorado de Planificación Estratégica, Calidad y Responsabilidad Social ${ }^{10}$ y que tiene por objetivo fundamental sensibilizar a la población universitaria en materia de derechos humanos, a través de actividades de difusión y promoción sobre ellos, entre los que como actividad sobresaliente, se encuentra, la participación en el Concurso de simulación judicial ante la CPI.

Lógicamente esta tarea también ha procurado un alto conocimiento técnico a los participantes. Conocimientos que exceden de manera notable a los que otros estudiantes, en idéntico nivel de formación, presentan, generalmente, sobre los mismos.

Ha sido necesario también aprender a controlar y gestionar la carga de estrés que intrínsecamente presenta una actividad como la apuntada. Considérese que implica entre otras cuestiones, un arduo trabajo de preparación académica (investigación), trabajo personal de cada uno de los participantes, y también completar una compleja disposición logística, necesaria para alcanzar el objetivo.

\section{RELEVANCIA Y REPERCUSIÓN}

Es de reputar como muy relevante la participación del equipo que representó a la Universidad de Jaén, sobre todo para responder al objetivo principal a perseguir, promover la Cultura de los Derechos humanos en la Universidad de Jaén. Sin embargo, no se agota aquí la relevancia y repercusión de la actividad y que repercute en diferentes actores:

a) Relevancia para el Proyecto "Semillero de sensibilización, investigación y cooperación jurídica en materia de Derechos Humanos”.

La actividad presentada constituye la acción más relevante proyectada desde el proyecto Semillero de cara a la consecución de su objetivo: promover la Cultura de los Derechos humanos en la Universidad de Jaén. El alumnado involucrado ha debido preparar

\footnotetext{
${ }^{9}$ Proyecto dirigido por el Prof. Dr. Víctor L. Gutiérrez Castillo.

${ }^{10}$ Sus funciones han sido asumidas por el Vicerrectorado de Estrategia y Gestión del Cambio de la Universidad de Jaén.
} 
concienzudamente el caso propuesto en el Concurso CPI y han alcanzado un notable conocimiento de los mecanismos de protección internacionales en materia de tutela jurisdiccional de los Derechos humanos.

b) Relevancia para la Universidad de Jaén

La Universidad de Jaén ha sido representada en un foro internacional excepcional. Se recuerda se trata de un Concurso internacional abierto a la participación de todas las universidades. Además mediante el empleo de la imagen corporativa de la Universidad, pensamos haber contribuido a dar a conocer la Universidad de Jaén en un foro internacional.

c) Relevancia para los participantes.

Los participantes demuestran haber incrementado sus aptitudes jurídicas en ámbitos de investigación técnica. Han demostrado su capacidad para elaborar dictámenes jurídicos, de la entidad requerida para ser presentados ante la CPI. Además han trabajado e implementado otras actividades, tales como trabajar bajo presión, control de oratoria, capacidad de hablar en público, control, liderazgo, etc.

d) Relevancia para el profesor asistente.

El profesor asistente considera haber aumentado considerablemente sus competencias a través de la actividad promovida y que ha preparado en exclusiva con el alumnado implicado. Ha debido gestionar y coordinar al grupo, tanto académica como logísticamente, en ésta y en otras actividades llevadas a cabo desde el Proyecto Semillero. Por lo que reputa como muy satisfactoria y positiva la actividad llevada a cabo. Además dicha actividad le ha permitido incrementar sus conocimientos en torno a las jurisdicciones internacionales y en particular sobre el proceso ante la CPI, entre otros muchos aspectos que gracias a esta actividad ha podido conocer, o implementar.

e) Relevancia para el resto de alumnado.

Es conocida por parte del alumnado de la Universidad de Jaén la participación de la Universidad en este concurso, a través de las actividades de difusión realizadas. Ello ha hecho que crezca el interés sobre estos concursos. El interés ha llegado al punto en que los alumnos de los Grados aludidos han manifestado su intención de participar en la preparación de la convocatoria de simulación judicial CPI en 2020. A estos efectos se propone completar la actividad representando el proceso que se desarrolló en La Haya, en la Universidad de Jaén, actividad que estará enfocada al alumnado de los Grados de Derecho y Doble Grado de Derecho y Administración de Empresas, siempre que hayan cursado, o lo estén haciendo, la asignatura de Derecho Internacional Público.

\section{CONCLUSIONES}

A partir de este ensayo se ha pretendido reflejar por extracto cuál ha sido el proceso de preparación y aprendizaje del grupo de alumnos/as que ha compuesto al equipo que ha representado a la Universidad de Jaén en el concurso de Simulación judicial ante la CPI, en su edición de 2019.

La primera premisa a exponer como conclusión es la relativa a que dicha actividad ha supuesto un muy importante reactivo para promover en la Universidad de Jaén la Cultura de los Derechos humanos. Ello a través de un mecanismo internacional, de naturaleza jurisdiccional, previsto para su salvaguarda. 
También se considera haber alumbrado el camino al alumnado de la Universidad de Jaén por cuanto respecta a posibles expectativas laborales en el ámbito de la Función Pública Internacional y haber incrementado al mismo tiempo las capacidades jurídico-prácticas que deben ser favorecidas desde la Universidad como complemento a los estudios de Grado señalados.

Se pone fin a este trabajo afirmando que la actividad realizada ha reportado muy grandes beneficios a los participantes que la han llevado a cabo, coincidiendo todos/as en calificarla como única.

\section{BIBLIOGRAFÍA}

Ambos, K., (1998) "El camino desde los primeros procesos por crímenes de guerra hasta la creación de la Corte Penal Internacional”, Historia de los derechos fundamentales, (coords.) Ansuátegui, F. J., Rodríguez Uribes, J. M., Peces-Barba Martínez, G., y Fernández García, E., vol. 4, Tomo 3, 1998, (El Derecho internacional de los derechos humanos), pp. 1697-1805.

Annello, C. S., (2013), “Desafíos de la Corte Penal Internacional en torno a la cooperación con los Estados”, Anuario Iberoamericano de Derecho Internacional Penal, vol. 1, nº 1 (Anuario Iberoamericano de Derecho Internacional Penal), pp. 43-60

Eizaguirre, A., y García Feijoo, Ma , (2004) "El coaching: una herramienta innovadora para el desarrollo del futuro licenciado. Estudio de un caso real de puesta en marcha del proceso de coaching con alumnos universitarios”, Pedagogía universitaria, hacia un espacio de aprendizaje compartido: III Simposio Iberoamericano de Docencia Universitaria, vol. 1, pp. 657-678.

Font Ribas, A. y Caballol i Angelats, L., (2018) "Simulación de un juicio como TFG”, REDU: Revista de Docencia Universitaria, vol. 16, $\mathrm{n}^{\circ} .2$.

Olásolo Alonso, H., Martínez Pascual, J. R., y Rodríguez, Ma S., (2016) "La inmunidad de jurisdicción penal por crímenes internacionales de los jefes de estado, los jefes de gobierno y los ministros de asuntos exteriores”, Revista chilena de derecho, vol. 43, nº 1 , pp. 251281.

Pérez González, M., (dir.), Conde Pérez, E., (coord.), (2012), Lucha contra el terrorismo, Derecho Internacional Humanitario y Derecho Penal Internacional, Tirant lo Blanch, Valencia. Vallejo Peña, C., (2016), El estado de la jurisdicción universal en el Derecho internacional y en el Derecho interno español, Tirant lo Blanch, Valencia.

\section{Normativa:}

Acuerdo de relación entre la Corte Penal Internacional y las Naciones Unidas, Nueva York, 4 de octubre de 2004. En vigor 04 de octubre de 2004.

Reglas de Procedimiento y Prueba de la CPI.

Reglamento de la CPI (2004).

Instrumento de Ratificación del Estatuto de Roma de la Corte Penal Internacional, hecho en Roma el 17 de julio de 1998. Disponible en BOE, núm. 126, de 27 de mayo de 2002, páginas 18824 a 18860 . 


\section{Otros documentos:}

Convocatoria Concurso CPI VII edición del Concurso CPI de Simulación Judicial ante la Corte Penal Internacional.

Guía para participantes en el VII Concurso CPI de Simulación Judicial ante la Corte Penal Internacional (2019).

Video de la final de la VII Edición del Concurso CPI Simulación Judicial ante la Corte Penal Internacional. Disponible a través del enlace: https://www.youtube.com/watch?v=DQWMljVa6yc.

Web Proyecto COPLA: https://www.coalicioncopla.org/. 